\title{
Мониторинг и прогноз развития эпидемии COVID-19 в г. Москва: выбор моделей на основе технологии сбалансированной идентификации (сентябрь 2021)
}

\author{
Соколов Александр Витальевич ${ }^{1}$, Соколова Любовь Александровна ${ }^{2}$ \\ ${ }^{1}$ Институт проблем передачи информации им. А.А.Харкевича РАН, г. Москва, Россия \\ alexander.v.sokolov@gmail.com \\ ${ }^{2}$ Институт системного анализа Федерального исследовательского иеетра \\ «Информатика и управление» РАН, г. Москва, Россия \\ las.sokolova@gmail.com
}

\begin{abstract}
Аннотация. Любая математическая модель является отражением знаний об исследуемом объекте. В работе показано, как накопление информации (статистических данных и знаний) о пандемии COVID-19 приводит к уточнению математических моделей, к расширению области их использования. Построенная в результате модель удовлетворительно описывает динамику заболеваемости COVID-19 в г. Москва с 19.03.2020 по 01.09.2021 и может использоваться для прогноза с горизонтом в несколько месяцев. Основным внутренним механизмом, определяющим динамику модели, является коллективный иммунитет. Мониторинг ситуации в г. Москва пока (до 01.09.2021) не выявил заметной сезонности заболевания и заметного повышения заразности (из-за появления штамма Дельта). Приводятся результаты использования технологии сбалансированной идентификации для мониторинга пандемии COVID-19:

- модели, соответствующие данным, доступным в различные моменты времени (с марта 2020 по август 2021);

- полученные новые знания (зависимости);

- прогнозы третьей и четвертой волны в г. Москва.
\end{abstract}

Обсуждаются трудности, возникшие после 01.09.2021, и направления дальнейшего модифицирования модели.

Ключевые слова: моделирование, мониторинг, прогноз, COVID-19, сбалансированная идентификация

\section{Введение}

Эффективное проведение мониторинга состояния и прогноза поведения сложных объектов подразумевает широкое использование математического моделирования. При определении целей мониторинга и прогноза необходимо учитывать наличие математических моделей, сложность, подробность и надежность которых в свою очередь определяются количеством и качеством (точностью) экспериментальных данных и знаний о функционировании объекта.

В случае пандемии COVID-19 по мере накопления данных и знаний и построения все более подробных и точных моделей, возможности мониторинга и прогноза возрастают. В доступных рядах данных проявляются различные эффекты, что позволяет усложнять соответствующие математические модели. Такому усложнению способствует и появление новых знаний об исследуемых процессах. 
Динамика эпидемии определяется процессами взаимодействия вируса, человеческого организма и общества. Различные процессы имеют различные характерные времена, причем, чем больше характерное время процесса, тем больше времени требуется для его проявления, тем длиннее должны быть ряды наблюдений, необходимые для определения его характеристик.

Для пандемии COVID-19 характерные времена различных процессов могут быть оценены следующим образом:

- 15 дней - заразность и манифестация (выявляемость) как функции длительности заболевания;

- 30 дней - текущий индекс репродукции (контактное число) и индекс выявления (и последующей изоляции);

- 60 дней - зависимость индекса выявления от количества проведенных тестов;

- 90 дней - влияние естественного (после болезни) коллективного иммунитета;

- 200 дней - влияние ослабления естественного иммунитета со временем;

- 100 дней - влияние вакцинации;

- 180 дней (предварительная оценка) - влияние ослабления искусственного (после вакцинации) иммунитета,

- 365 дней - сезонность заразности

Горизонт прогноза определяется погрешностью модели, которая определяется точностью описания отдельных процессов, которая в свою очередь определяется длинной рядов наблюдений (и их надежностью). Таким образом длительность мониторинга определяет какие процессы могут быть оценены (с характерными временами меньше этой длительности) и, следовательно, определяют горизонт и точность прогноза. С этой точки зрения становится понятной неудача попытки прогноза динамики пандемии, предпринятой Н. Фергюсоном. Его группа в начале 2020 года активно обосновывала тотальный карантин, "прогнозируя" (на несколько месяцев вперед) к лету 2020 года 500 тыс. смертей в Англии и 2 млн. в США (за лето 2020 года) [https://www.imperial.ac.uk/media/imperial-college/medicine/mrc-gida/2020-0316-COVID19-Report-9.pdf, стр. 7].

На начальном этапе (первые месяцы пандемии), когда объем знаний и статистических данных был невелик и ненадежен, мы ограничились целью поиска закономерностей взаимодействия вируса и человека (заразность и манифестация вируса как функция длительности заболевания) и описания некоторых социальных механизмов управления эпидемией (количество контактов и изоляция зараженных). Рассматривались процессы с характерными временами около 30 дней.

Затем, по мере накопления опыта (анализ траекторий модели и сравнение их с данными), знаний и статистической информации, был проведен ряд модификаций, позволивших учесть более медленные процессы (с большими характерными временами):

- эффективность изоляции зараженных в виде функции от количества проводимых (ежедневно) тестов (new tests) (лето 2020);

- естественный иммунитет переболевших (осень 2020);

- потерю иммунитета в виде функции, зависящей от времени, прошедшего с момента заражения (зима 2020-2021);

- вакцинацию (весна-лето 2021) 


\section{Источники данных.}

При построении моделей использовалась следующая статистическая информация:

- Новые случаи заражения в Москве - из официальной отчетности (см., например, https://стопкоронавирус.pф/information/).

- Количество проводимых тестов в России - из официальной отчетности (см., например, https://tatspravka.ru/statistika-russia/) Количество проведенных тестов на 1000 чел. в Москве оценивалось в 1/5 от проведенных в России.

- Процент носителей антител (имеющих иммунитет) в Москве - из заявлений официальных лиц и цитировании в интернете:

- https://ria.ru/20200710/1574177822.html,

- https://www.vedomosti.ru/society/news/2020/09/28/841417-popova-nazvala-dolyu-moskvicheis-antitelami-k-koronavirusu,

- https://www.vesti.ru/article/2533510

- Количество проведенных в Москве прививок - из официальной отчетности (см., например, https://стопкоронавирус.pф/news/)

\section{Методы и модели}

Для выбора моделей, соответствующих количеству и качеству данных, использовался метод сбалансированной идентификации [Sokolov 2020a] и одноименная информационная технология, созданная в Центре распределенных вычислений Института Проблем Передачи Информации РАН. В настоящий момент доступна программная реализация технологии (https://github.com/distcomp/SvF).

Следует отметить, что основная переменная модели, количество невыявленных инфицированных, не является наблюдаемой (измеряемой) величиной. Наблюдаемой величиной являются новые случаи заражения $(n C)$, которые являются неизвестной функцией от невыявленных. Это приводит к неоднозначности идентификации - внутренняя динамика модели определяется с точностью до множителя. Для преодоления этого недостатка в критерий сбалансированной идентификации добавляется специальное слагаемое, минимизирующее полное количество зараженных. Кроме того, для восстановления корректности постановки задачи (устранения неоднозначности идентификации) используются дополнительные данные - количества носителей антител, представляющие собой интегралы от полного количества инфицированных, имеющих иммунитет.

В терминах принятых обозначений эпидемиологических моделей (см., напр. [Brauer 2019]) используются различные модификации моделей SIR и SIRS, отличительной особенностью которых является разбиение инфицированных $(I)$ на 15 групп, в соответствии с длительностью заболевания (Age of Infection Model, см. например, $S I_{15} R$ в Приложении). В терминах моделей популяционной динамики и демографии используются (матричные) модели Лесли [Свирежев 1978] и модели Маккендрика фон Фёрстера (McKendrick Von Foerster) [Nakhushev 1995, Эбелинг 2001].

Структура моделей изменялась по мере доступности новых данных и учета новых процессов (тестирование, вакцинация и т.п.). Специфические "символьно-числовые" обозначения соответствующих модификаций отражают используемые данные и учитываемые процессы. Формальные описания моделей приведены в приложении. 


\section{Результаты}

В начале пандемии мы не знали о вирусе почти ничего, а доступная статистическая информация ограничивалась числами новых случаев заражения $(n C)$ и смерти. На этом этапе мы ограничились целью выявления закономерностей взаимодействия вируса и человека заразности и манифестации в зависимости от длительности болезни. Для этого использовалась модель динамики невыявленных зараженных $S I_{15} R$ - $n C$ (см. Приложение), распределенных по длительности заражения. В качестве данных использовались только временные ряды выявленных зараженных $(n C)$. Уже через пару месяцев на основе обработки временных рядов по 7-ми популяциям (Великобритания, Германия, Италия, Испания, Франция, Россия без г.Москвы и Московской области и г.Москва с Московской областью) удалось оценить функции взаимодействия человека и вируса, определяющие динамику эпидемии, например, заразность как функцию от длительности (времени) болезни (см. рис 1), а также оценить социальные управления - текущий индекс репродукции $R O_{t}$ (контактное число) и индекс выявления (и последующей изоляции) $I S_{t}$. Подробно с результатами можно ознакомиться в [Sokolov 2020].

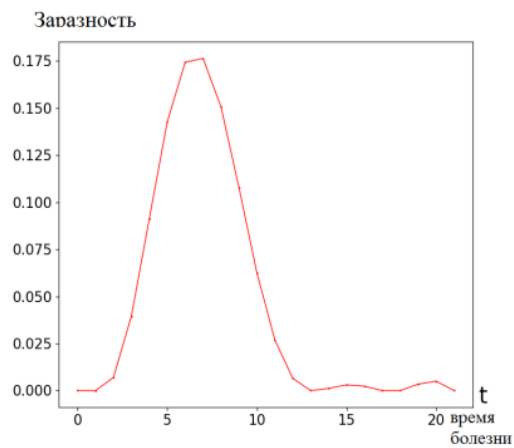

Рис.1. Нормированная (интеграл равен единице) заразность как функция от длительности заражения (дни).

К лету 2020 г. накопился достаточный объем новой информации — число проведенных тестов. Это позволило поставить новую цель - описать усилия общества по выявлению (и последующей изоляции) больных в зависимости от количества проведенных тестов. Соответствующая модифицированная модель $S I_{15} R-n C$ - $n T$ (см. Приложение) позволила оценить связь количества выявленных зараженных с количеством проведенных тестов. Найденная функция (с учетом бессимптомных и незарегистрированных носителей вируса) эффективности выявления в зависимости от количества тестов на тысячу человек для Москвы приведена на рис. 2. Анализ кривой позволяет для данной модели оценить эффективность выявления больных в Москве величеной не более 17\%, т.е. из полного числа инфицированных выявляется и изолируется не более одного человека из шести.

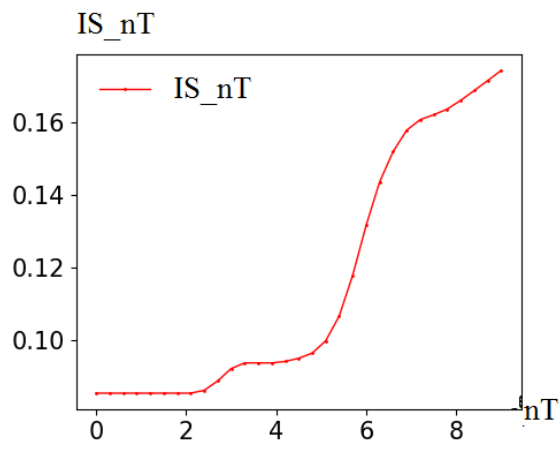

Рис. 2. $I S \_n T-$ функция эффективности выявления больных в зависимости от количества тестов на тысячу человек $(n T)$ в г. Москва. 
Рассмотренная модификация уже может быть использована для прогноза заболеваемости при различных сценариях интенсивности тестирования.

Рассмотренные две модели были линейными, они определяли внутреннюю динамику с точностью до множителя и не учитывали размер популяции. Реальное количество инфицированных неизвестно (не является наблюдаемой величиной). Для оценки полного числа инфицированных нужна информация нового типа.

Летом 2020г. такая информация появилась - (скрининговые) оценки числа носителей (значимого титра) антител, представляющие собой интеграл от полного количества переболевших (а не только выявленных). Для Москвы эта (не систематическая) информация «добывалась» из заявлений официальных лиц. Соответствующая модификация модели $S I_{15} R$ $n C$-nT-Anti позволила для Москвы оценить реальное количество зараженных (не только выявленных), учесть количество переболевших (считается, что у них имеется естественный иммунитет), что существенно улучшило прогностические возможности модели. Модель престала быть линейной, появилось саморегулирование процесса.

Однако, в декабре 2020г. пространство решений модели стало недостаточно емким для описания наблюдаемых данных, что вызвало очередную ее модификацию - учет потери естественного (полученного в результате заболевания) иммунитета. Идентификация новой модификации модели $S I_{15} R S$-nC-nT-Anti-Im позволила оценить время утраты иммунитета в 190 дней (в среднем) - соответствующая кривая приведена на рис 3.

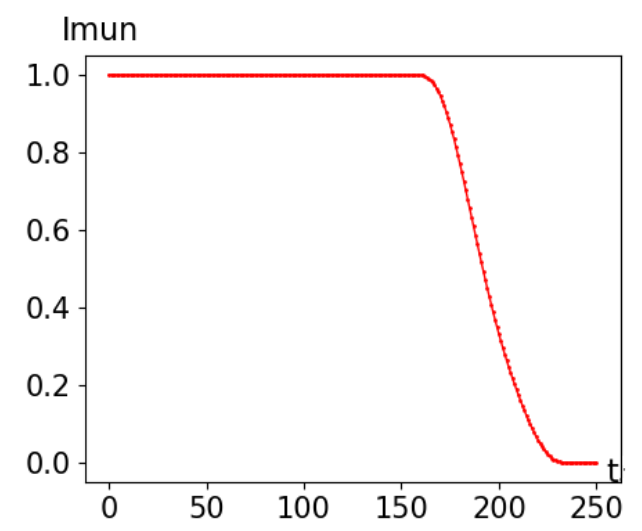

Рис. 3. Сохранение естественного иммунитета как функция времени от начала заболевания (дни). 1 - полный иммунитет, 0 - отсутствие иммунитета.

Наконец, с начала 2021 г. началась массовая вакцинация. Для ее учета в модификации модели $S I_{15} R S$-nC-nT-Anti-Im-Vuc добавлен множитель, уменьшающий количество новых инфицированных в зависимости от процента вакцинированных. В этой модификации для управления эпидемией имеется уже три средства (социального) управления: вакцинация, тестирование и ограничение контактов.

\section{Прогнозы}

К декабрю 2020 накопился достаточный опыт мониторинга, и основные процессы, описывающие динамику пандемии, были выявлены, описаны и идентифицированы. Построенные в результате модели использовались для прогнозирования.

На рисунках 4, 5, 7, 8 и 12 приведены результаты моделирования, идентификации, прогноза и верификации новых (выявленных) случаев. На оси X откладывается количество дней от начала моделирования - 19.03.2020. Для наглядности над осью X нанесены отметки, 
соответствующие месяцам и годам. Вертикальная желтая линия соответствует дате начала прогноза. Модельные кривые (красного цвета) были получены (идентифицированы) на обучающем наборе данных (синие точки). Построенная таким образом модель использовалась для прогноза. При этом социальные управления модели (индекс репликации, количество новых тестов и количество новых вакцинированных), как правило. замораживались на последнем (перед прогнозом) значении. Для верификации модели использовался полученный позже и незадействованный при построении модели набор данных (зеленые точки). Сравнение прогноза (красной кривой) с тестирующим набором показывает в течение нескольких месяцев неплохое совпадение. Возникающие расхождения, как правило, объясняются существенными изменениями социальных управлений.

\section{Прогноз от 01.12.2020 - конец второй волны}

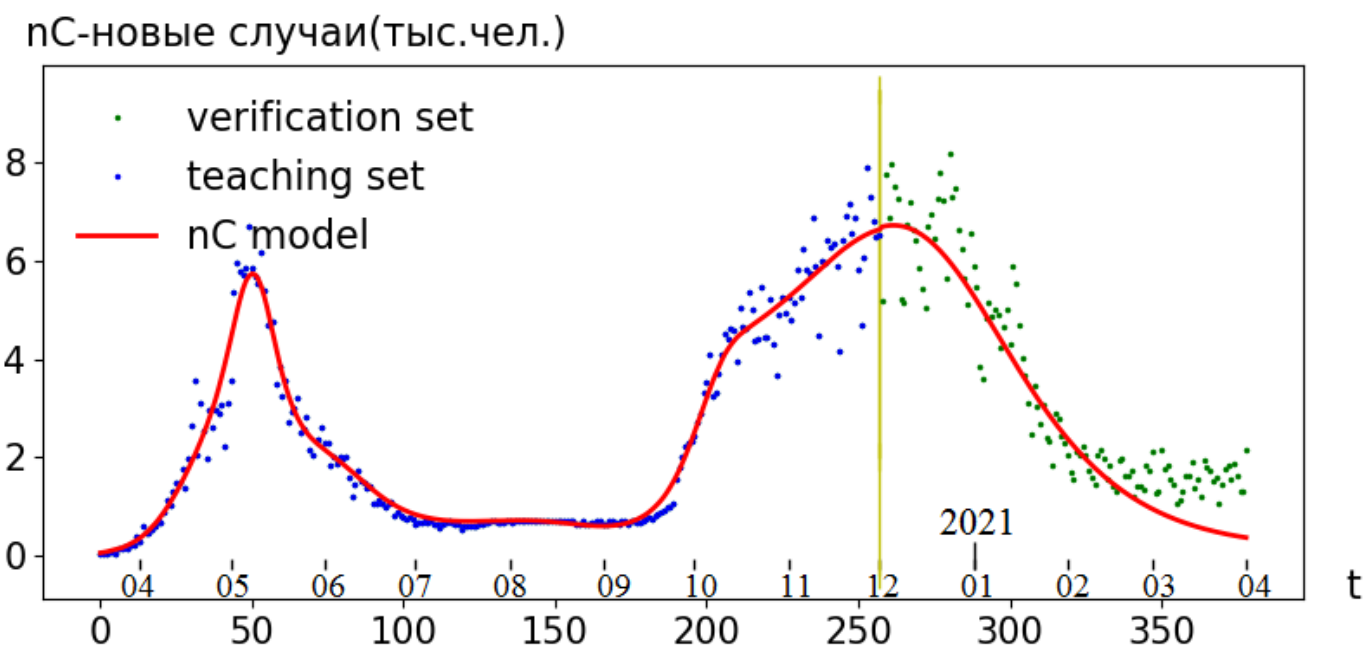

Рис.4. Прогноз новых случаев заражения в Москве от 01.12.2020. Синие точки - обучающий набор, красная кривая - модель, зеленые точки - тестирующий набор.

Согласно модели, падение числа выявленных случаев во второй волне (декабрь-январь 2021г.) в большой степени определяется накоплением иммунной прослойки (населения, имеющего естественный иммунитет). Наблюдаемое с середины февраля расхождение можно объяснить существенным ослаблением (противоэпидемических) ограничений и/или миграцией заболевших из других регионов России

\section{Прогноз от 15.04.2021 - третья волна}

На рис. 5. приведен пример прогноза третьей волны в Москве при заданных управлениях: контакты и тесты замораживаются на значениях. 2.43 и 4.48 соответственно (от 15 апреля 2021г), вакцинация - 12000 чел./день, эффективность вакцинации 91,6\% (см. рис. 
$6)$.

\section{nC-новые случаи(тыс.чел.)}

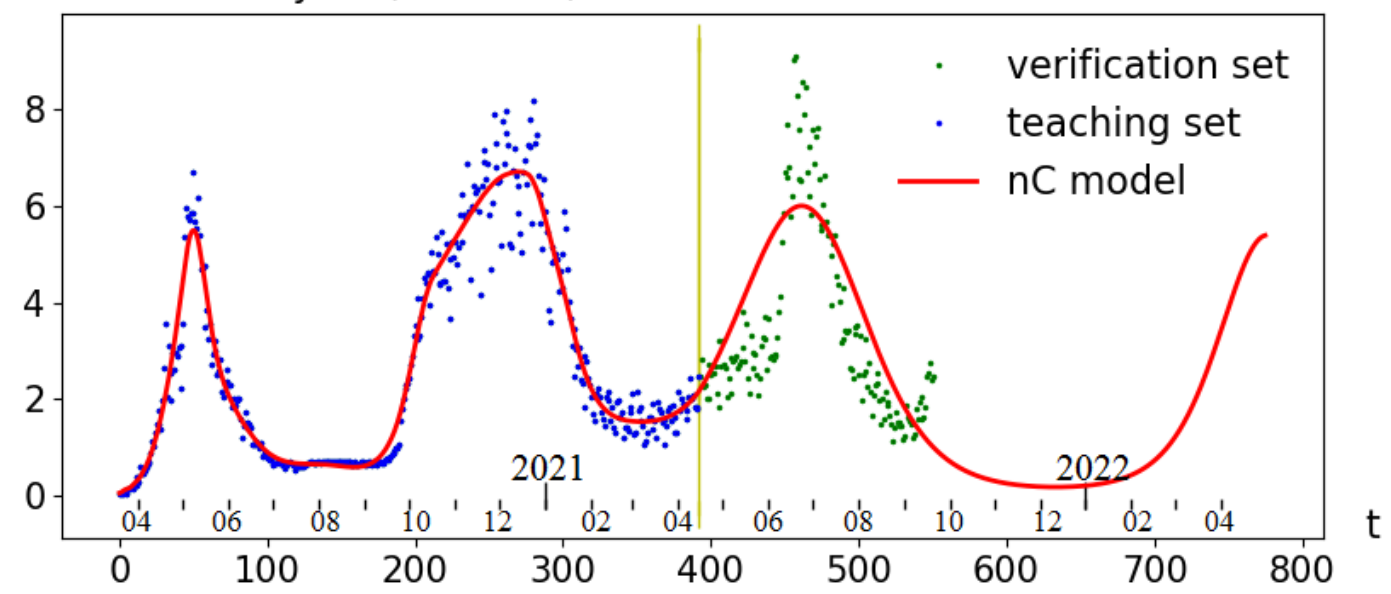

Рис. 5. Прогноз третьей волны эпидемии в Москве от 15.04.2021- новые случаи заражения. Синие точки - обучающий набор, красная кривая - модель, зеленые точки (верификация?) тестирующий набор (до 20.09.2021).

В течение последующих пяти месяцев прогноз качественно совпадает с реальными данными, которые не использовались при идентификации модели (зеленые точки). Согласно модели, третья волна (увеличение числа выявленных случаев с мая 2021г.) в большой степени определяется уменьшением иммунной прослойки переболевшего населения. Действительно, с мая месяца начинают терять иммунитет переболевшие в начале второй волны (октябрьноябрь). В июне рост выявленных зараженных резко усиливается, что соответствует потере естественного иммунитета значительного количества переболевших в декабре 2020. Периодичность волн составляет около 200 дней и, в основном, определяется функцией сохранения иммунитета (рис.3). Интенсивные мероприятия по временному уменьшению индекса репликации и увеличению тестирования (и последующей изоляции) могут несколько сдвинуть волну или изменить ее форму. При этом суммарное количество инфицированных меняется несущественно - основным механизмом, определяющим динамику, является общий (переболевших и вакцинированных) коллективный иммунитет. Согласно модели $\left(S I_{15} R S-n C\right.$ $n T$-Anti-Im-Vuc), для того чтобы волна пошла на спад, общий коллективный иммунитет должен превысить некоторое значение, которое определяется количеством тестов и индексом репродукции (ограничительными мерами общества). Для первой волны это значение составляло около $15 \%$ населения, для второй - около $35 \%$, для третей оценивается значением около $55 \%$.

При вакцинации 12 т./день прогноз количества выявленных зараженных в третьей волне (с 15.04.2021 по 01.11.2021) составляет 671 тыс. человек. Соответствующая оценка смертности (при условии, что умирают $2.5 \%$ от выявленных инфицированных) составляет около 16 тысяч. Введение (временных) ограничений, уменьшающих $R O_{t}$, и увеличение тестирования растягивает волну и уменьшает ее высоту, однако прогнозируемое суммарное количество выявленных зараженных в третьей волне меняется не существенно. Это некая «константа», которую можно существенно уменьшить лишь вакцинацией - по модельным расчетам (оценка на 17.06.2021), 11 вакцинированных уменьшают количество выявленных переболевших на 1. Таким образом, вакцинация дополнительных (по сравнению с прогнозом из расчета 12т./мес.) 1.5 мил. человек уменьшает кол-во выявленных заболевших на 135 т. При смертности $2.5 \%$ получаем, что 440 привитых уменьшают количество умерших в третьей 
волне на 1, соответственно 1.5 мил. дополнительных вакцинированных спасают около 3400 жизней.

Согласно официальной статистике, количество зараженных, выявленных в Москве с 15.04.2021 по 19.07.2001, составляет 410 т. чел., что неплохо согласуется с прогнозом в 671 т. чел., особенно, если принять во внимание уменьшение на 120 т. из-за непредусмотренного прогнозом интенсивного вакцинирования.

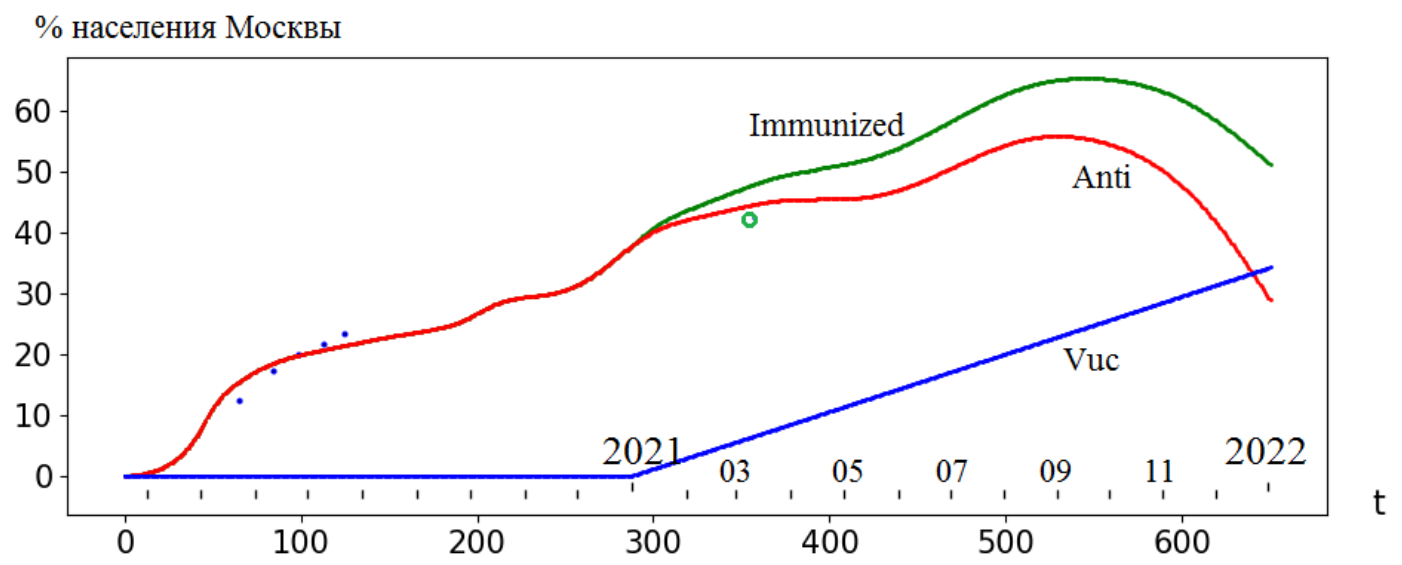

Рис. 6. Иммунизация населения Москвы. Vuc(t) - заданное управление процент вакцинированных (соответствует темпам вакцинации 12тыс./день). Anti(t) - прогноз процента переболевших, сохранивших к моменту $\mathrm{t}$ иммунитет. Immunized(t) - прогноз процента “невосприимчивых" (вакцинированных и переболевших с иммунитетом). Синие точки (процент носителей антител, т.е. переболевших) использовались при идентификации, точка, отмеченная зеленым кругом, оставлена для верификации.

На рис 6. приводится динамика коллективного иммунитета и его составляющих. Точка, отмеченная зеленым кругом (оценка процента переболевших, имеющих иммунитет на 08.03.2021) не использовалась при идентификации. Близость её к траектории можно рассматривать как дополнительное подтверждение пригодности модели для прогноза (верификация модели).

\section{Прогноз от 11.05.2021}

Представленный на рис. 7 прогноз (от 11.05.2021) соответствует уровню контактов, соответствующих праздничным дням (01.05.2021-10.05.2021), что и объясняет заметное замедление роста заболеваемости. В этом случае прогнозируемая 3 -я волна более низкая и широкая, но имеет почти такую же площадь (суммарное количество выявленных зараженных) как и в прогнозе на рис. 5. 


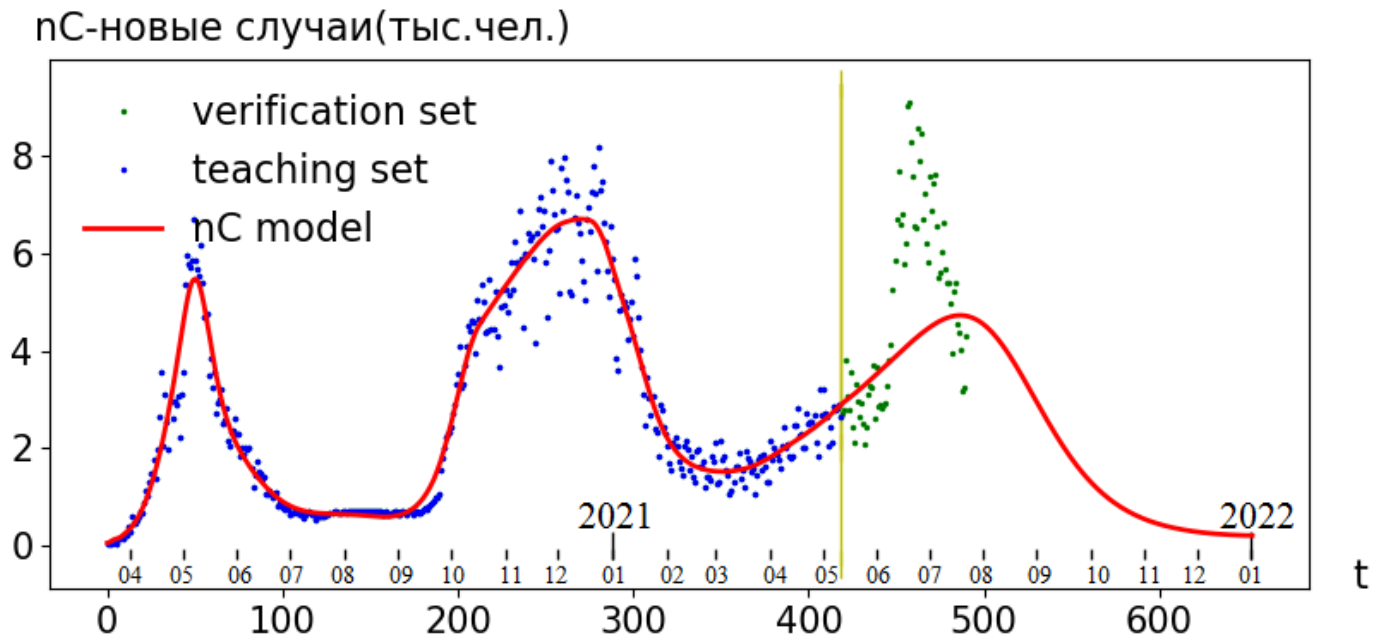

Рис. 7. Пример прогноза третьей волны в Москве от 11.05.2021.

\section{Прогноз от 25.07.2021- конец третьей волны}

Рассмотрим подробно. Расчет прогноза, приведенный на рис. 8, осуществлялся по следующим сценариям управления:

- Вакцинация в \% от населения Москвы (на рис. 9 график Vuc, сдвинутая на 2 недели) исходя из интенсивности 12 т.чел./день, эффективность равна 91.6,

- индекс репродукции (R0 на рис. 10) заморожен на последнем значении $\mathrm{R} 0=1.9$,

- $\quad$ количество тестов на тысячу населения (рис. 11) заморожено на последнем значении $\mathrm{nT}=8.7$.

Очевидно, что использованные сценарии (низкий индекс репродукции и значительное количество тестов) существенно занижают прогноз. В этом случае уже 50\% вакцинированных позволяют избежать четвертой волны (в конце 2021 г.).

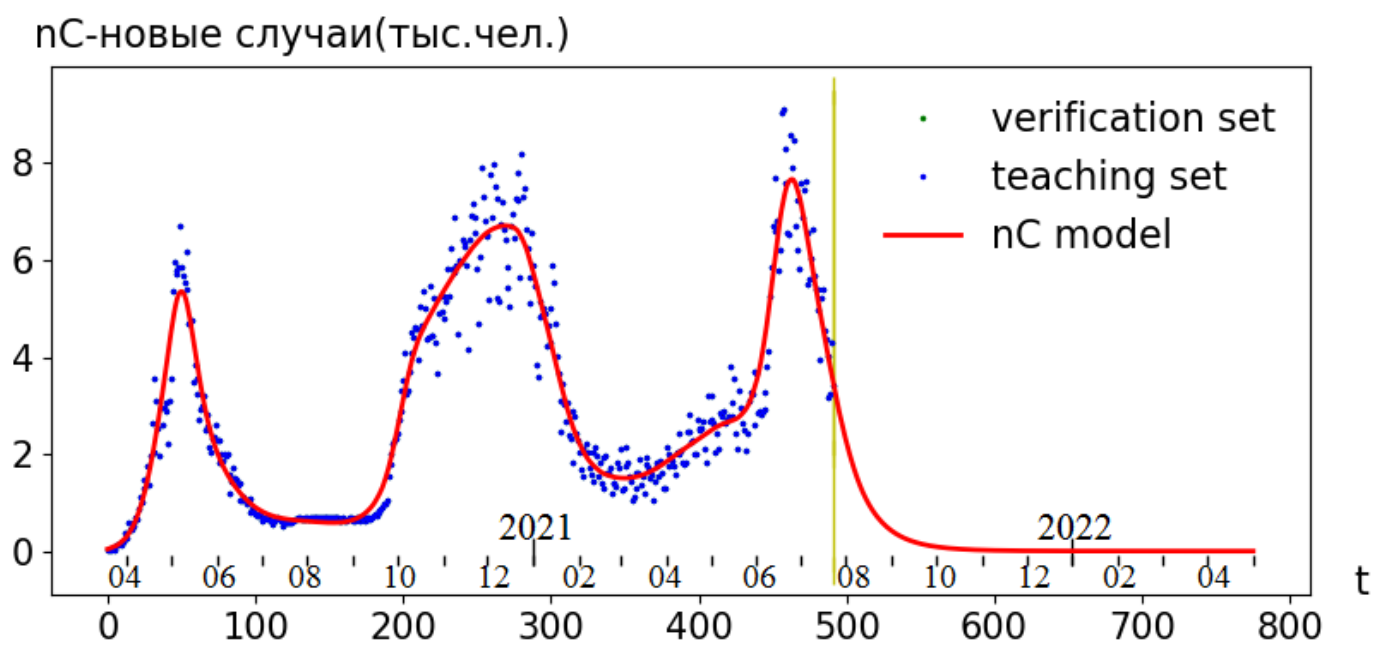

Рис. 8. Прогноз третьей волны в Москве от 25.07.2021. 


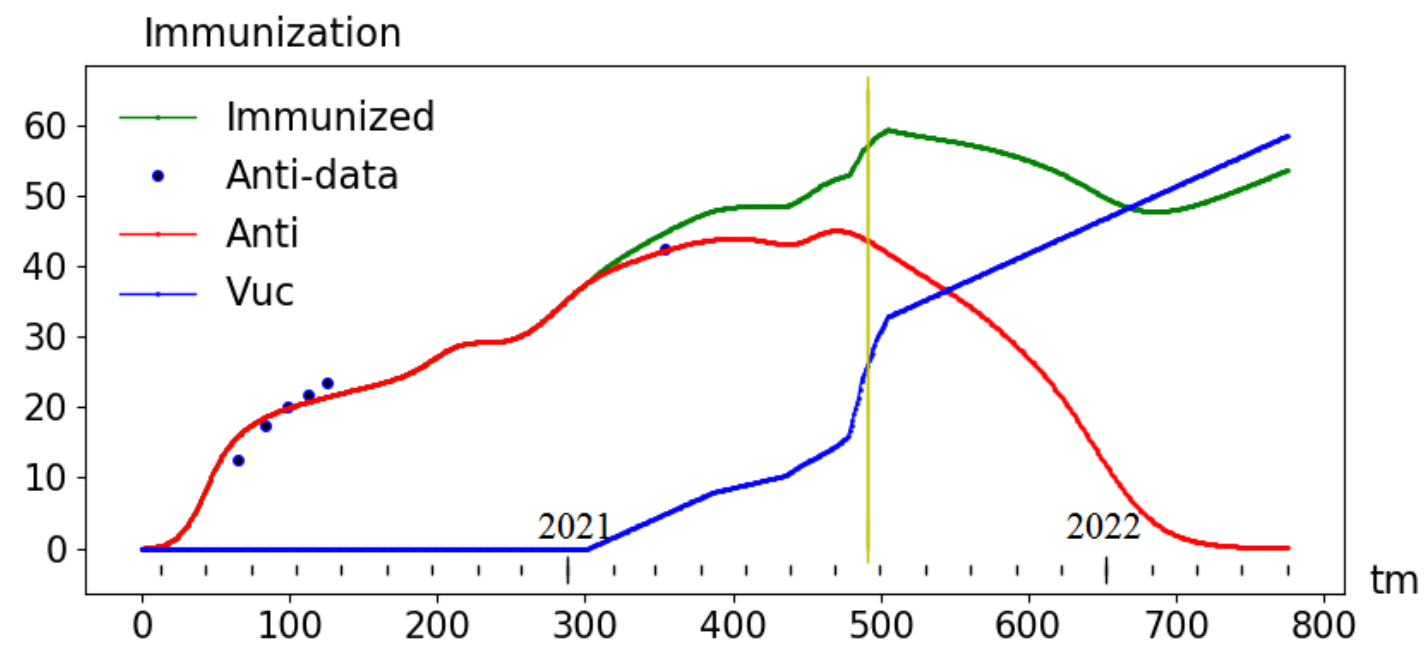

Рис. 9. Прогноз иммунизации населения в Москве от 25.07.2021. Vuc(t) - заданное управление процент вакцинированных (соответствует темпам вакцинации 12 тыс./день). Anti(t) - прогноз процента переболевших, сохранивших к моменту t иммунитет. Immunized(t) - прогноз процента "невосприимчивых" (вакцинированных и переболевших с иммунитетом).

\section{Индекс Репродукции}

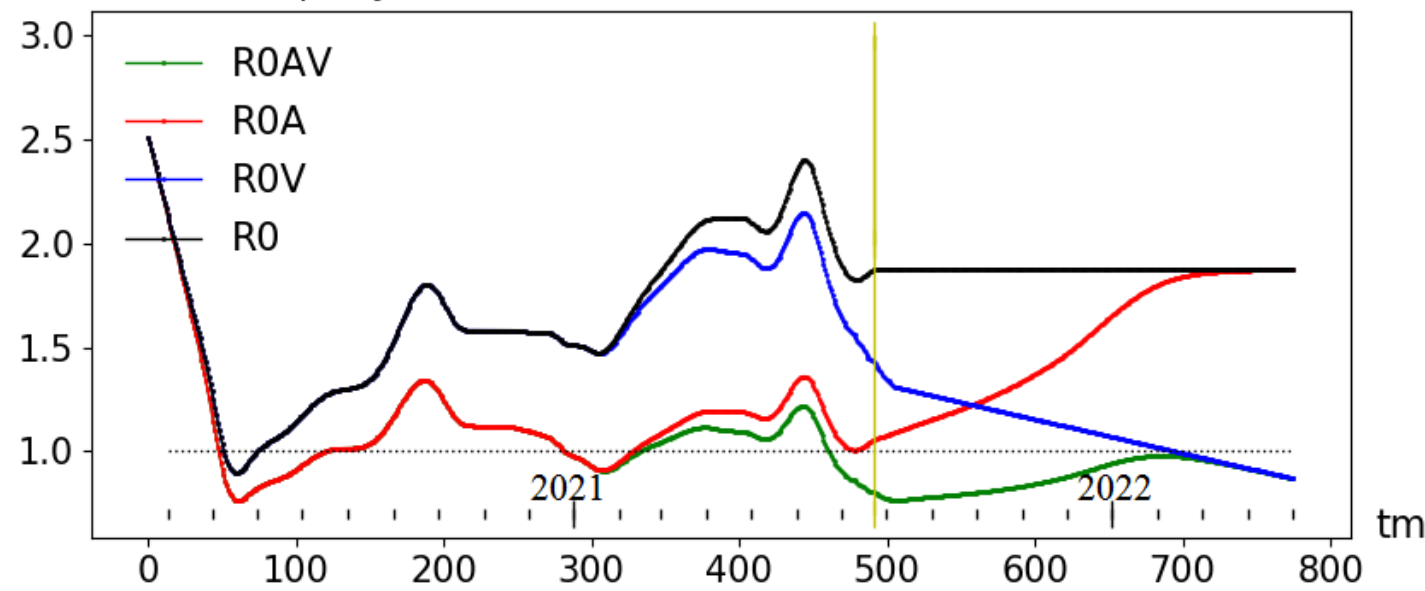

Рис 10. Индексы репродукции (от 25.07.2021): R0 - индекс репродукции (идентифицированная функция и заданное управление, справа от желтой линиии), R0A индекс репродукции с учетом иммунитета переболевших, R0V - индекс репродукции с учетом вакцинации, R0AV - индекс репродукции с учетом иммунитета переболевших и вакцинации. 


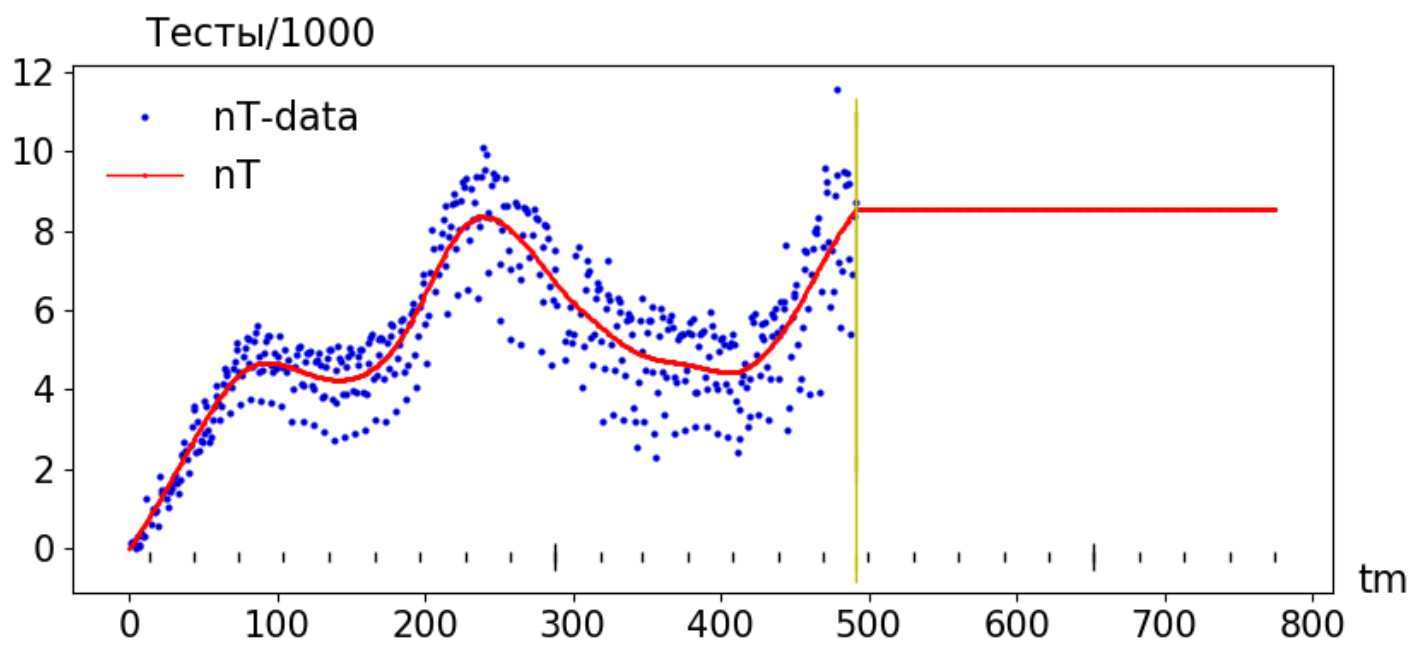

Рис. 11. Количество тестов на тысячу: аппроксимация данных и заданное управление (справа от желтой линии).

\section{Прогноз от 25.07.2021- четвертая волна}

На рис. 12-15 представлен более реалистический прогноз, который предполагает ослабление ограничений, уменьшение тестирования при сохранение старого сценария вакцинирования:

- Вакцинация в \% от населения Москвы (на рис. 13 график Vuc, сдвинутая на 2 недели) исходя из интенсивности 12 т.чел./день, эффективность равна 91.6,

- $\quad$ индекс репродукции (R0 на рис. 14) поднимается к началу сентября к значению 2.5 , которое соответствует отсутствию ограничений,

- количество тестов на тысячу населения (рис. 11) к ноябрю падает до умеренного значения 4.5.

- предполагается небольшой приток инфицированных извне г. Москвы. Их полное число составляет 600 чел./день, что увеличивает количество ежедневно выявляемых инфицированных на величину около 90 чел.

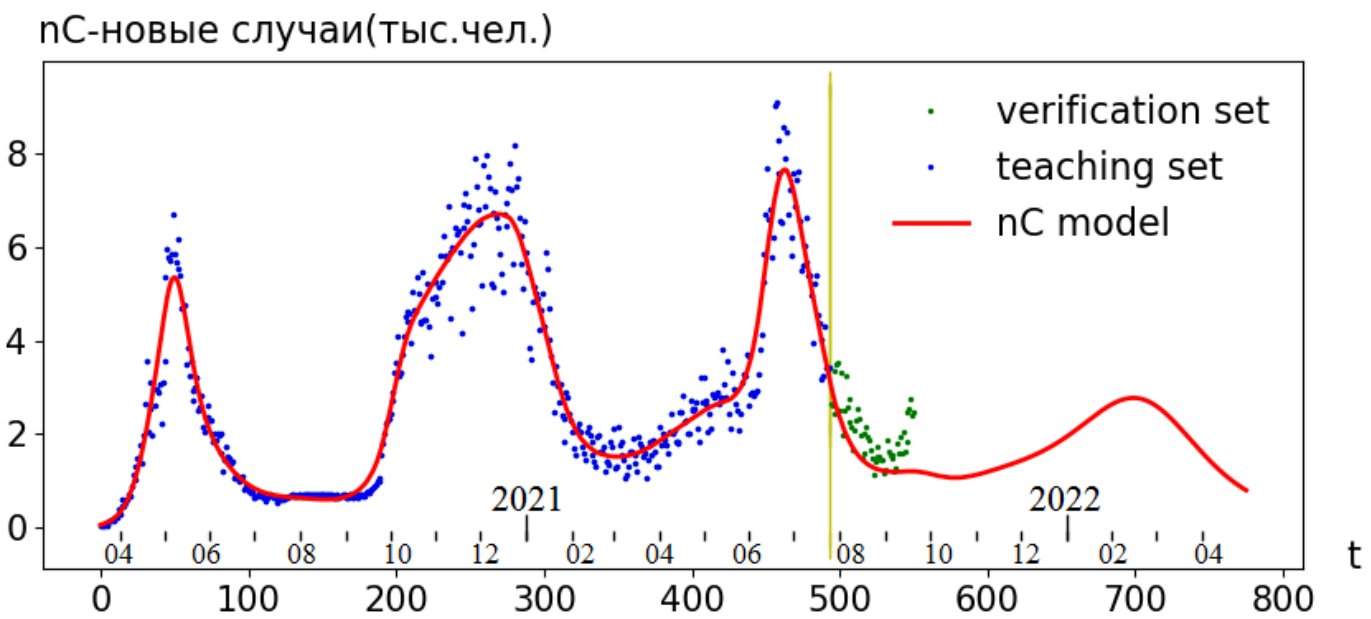

Рис. 12. . Прогноз четвертой волны в Москве от 25.07.2021 и его сравнение с реальными данными (зеленые точки) до 20.09.2021. 


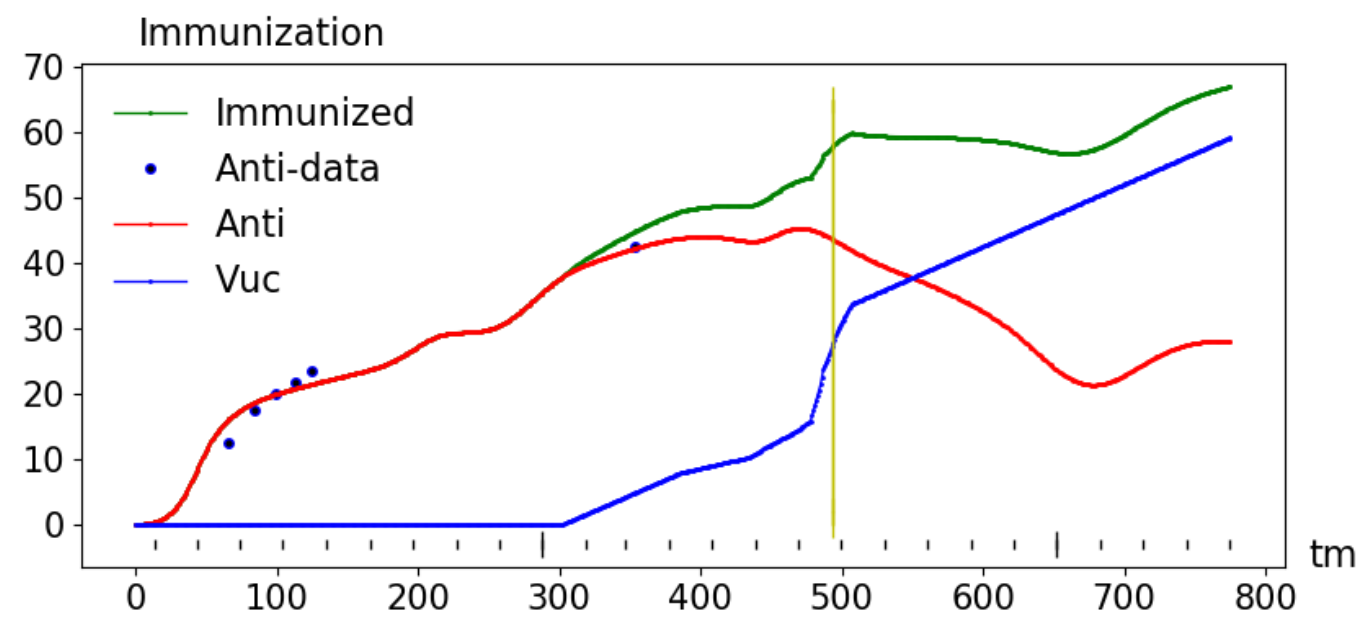

Рис. 13. Прогноз иммунизации населения Москвы в четвертой волне от 25.07.2021. Vuc(t) заданное управление процент вакцинированных (соответствует темпам вакцинации 12 тыс./день). Anti(t) - прогноз процента переболевших, сохранивших к моменту t иммунитет. Immunized(t) - прогноз процента “невосприимчивых" (вакцинированных и переболевших с иммунитетом).

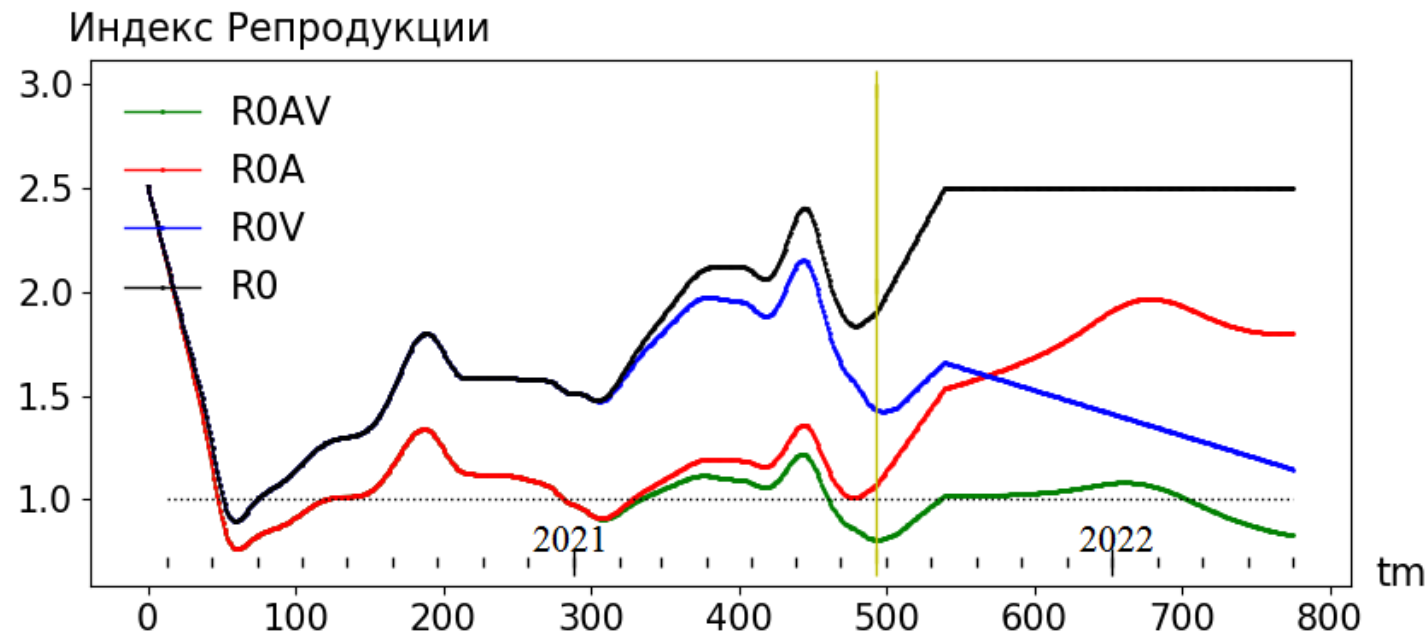

Рис. 14. Индексы репродукции (прогноз от 25.07.2021): R0 - индекс репродукции (идентифицированная функция и заданное управление, справа от желтой линиии), R0A индекс репродукции с учетом иммунитета переболевших, R0V - индекс репродукции с учетом вакцинации, R0AV - индекс репродукции с учетом иммунитета переболевших и вакцинации. 


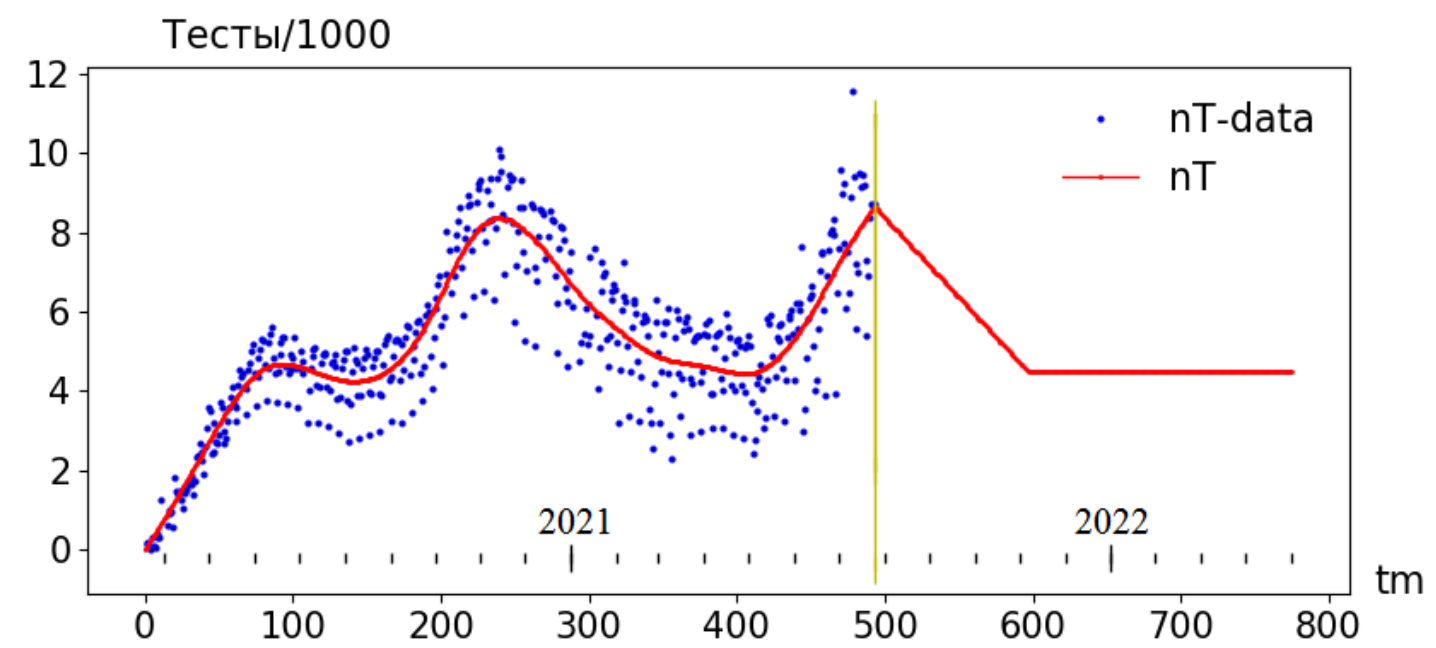

Рис. 15. . Количество тестов на тысячу: аппроксимация данных и заданный сценарий для прогноза четвертой волны от 25.07.2021 (справа от желтой линии).

На рис. 12 приведены прогноз новых случаев заражения и реальные данные. В течение первого месяца наблюдается неплохое совпадение, однако далее возникает заметное расхождение. Его можно объяснить существенным увеличением индекса репродукции из-за увеличения заразности (другой штамм) и/или уменьшения со временем иммунитета вакцинированных.

\section{Заключение.}

По мере наблюдения за пандемией COVID-19 в исследуемых рядах данных проявляются различные эффекты. Их учет позволяет последовательно (шаг за шагом) усложнять модель. Этот процесс не закончен -

В результате такого мониторинга удалось построить более или менее полную (на август 2021) модель, удовлетворительно описывающую весь годовой цикл эпидемии. Проявившиеся при прогнозах погрешности, как правило, объясняются неопределенностью сценариев (будущих) социальных управлений, необходимых для расчетов.

Построенная модель пока не учитывает:

- Ослабление иммунитета вакцинированных и ревакцинацию. К моменту подготовки публикации (август 2021) заметного проявления этого процесса в накопленных данных не обнаружено.

- Миграцию - влияние на заболеваемость в Москве заболеваемости в других регионах России и мире. Пока динамика выявленных инфицированных измерялась тысячами и определялась внутренними процессами, миграцией, по-видимому, можно было пренебречь. Однако массовая вакцинация должна существенно сократить число внутренних инфицированных (см. рис. 8 и 12) и увеличить удельный вес потока извне. Прогноз четвертой волны от 25.07.2021 с упрощенным учетом иммиграции продемонстрировал важность учета этого процесса.

- Существенного увеличения заразности, вызванной мутациями вируса.

В результате, динамика новых случаев заражения в сентябре месяце уже плохо “помещается" в используемую модель и требует очередной её модификации. Для этого придется подождать, когда во временных рядах (статистических данных) отразятся эффекты потери иммунитета от вакцинации, резкого увеличения заразность (штамм Дельта) и станет 
заметно влияние миграции. По-видимому, следующая существенная модификация модели станет возможна в октябре 2021.

Исследование выполнено при финансовой поддержке РФФИ в рамках научных проектов 20-57-82004 и 20-07-00701

\section{Литература}

- (Brauer 2019) Brauer F., Castillo-Chavez C., Feng Z., Mathematical Models in Epidemiology, 2019

- (Sokolov 2020) Sokolov A.V., Sokolova L.A., COVID-19 dynamic model: balanced identification of general biological and country specific features, 9th International Young Scientist Conference on Computational Science (YSC 2020), Procedia Computer Science, Volume 178, 2020, Pages 301-310, https://doi.org/10.1016/j.procs.2020.11.032

- (Sokolov 2020a) Sokolov A.V., Voloshinov V.V. Model Selection by Balanced Identification: the Interplay of Optimization and Distributed Computing, Open Computer Science, 2020, 10, p. 283-295. DOI: 10.1515/comp-2020-0116, https://doi.org/10.1515/comp-2020-0116

- (Свирежев 1978) Свирежев Ю.М., Логофет Д.О. Устойчивость биологических сообществ. - Москва: Наука, 1978, 352с.

- (Nakhushev 1995) Nakhushev A.M. (1995) The equations of mathematical biology. Textbook manual for universities. M .: Higher School: 301.

- (Эбелинг 2001) Эбелинг Вернер, Энгель Андреас, Файстель Райнер. Физика процессов эволюции. Пер. с нем. Ю. А. Данилова. - М.: Эдиториал УРСС, 2001. - 328 с.

\section{Приложение - Математические модели.}

$S I_{15} R$.

Разностная модель. Согласно рис. П1 в данной модели население разбивается на 3 основные группы: $\boldsymbol{S}$ - восприимчивые, $\boldsymbol{I}$ - инфицированные (невыявленные) и $\boldsymbol{R}$ невосприимчивые (удаленные: выздоровевшие и изолированные). Кроме того группа инфицированных разбивается на 15 подгрупп, в соответствии со временем, прошедшим с момента заражения $\boldsymbol{a}$ (age of infection).

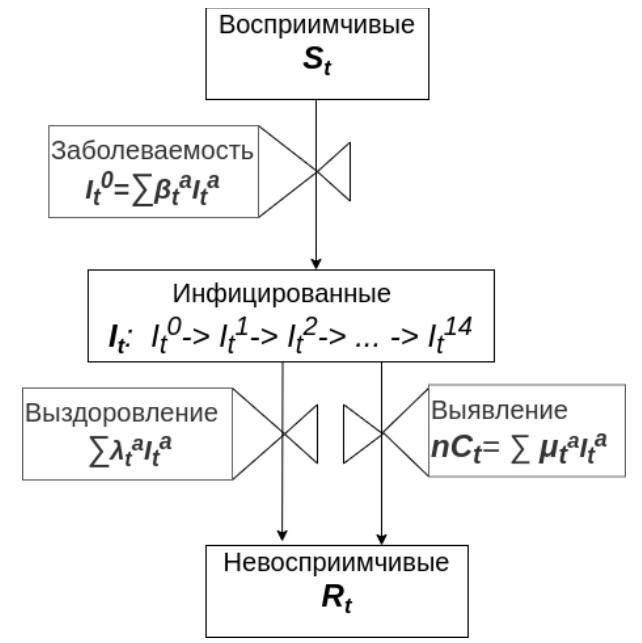

$A$

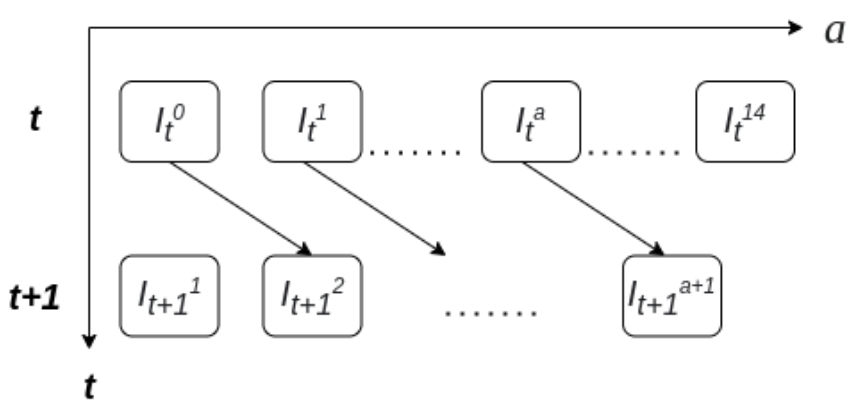

$B$ 
Рис. П1. А. Модель $S I_{15} R$ с разбиением инфицированных на 15 групп. $B$. Иллюстрация динамики групп инфицированных, которая определяется сдвигом по возрасту заболевания $(a)$.

Формализация, рассмотренной схемы приводит к разностной модели:

$$
\begin{aligned}
& I_{t+1}^{a+1}=I_{t}^{a}-\lambda^{a} \cdot I_{t}^{a}-\mu_{t}^{a} \cdot I_{t}^{a} ; a \in[0,14] ; t \in[0, T] \\
& I_{t+1}^{0}=B_{t} ; t \in[0, T] \\
& B_{t}=\sum_{a^{\prime}=0}^{14} \beta_{t}^{a^{\prime}} I_{t}^{a^{\prime}} ; t \in[0, T] \\
& I_{0}^{a}=I_{0}^{a} ; a \in[0,14] \\
& n C_{t}=\sum_{a^{\prime}=0}^{14} \mu_{t}^{a^{\prime}} I_{t}^{a^{\prime}} ; t \in[0, T]
\end{aligned}
$$

где $a$ - длительность (возраст) заболевания, $t$ - время моделирования. $\quad I_{t}^{a}-$ количество (невыявленных) зараженных в момент времени $t$ с длительностью заражения $a, \lambda^{a}$ - вероятность выздороветь, $\mu_{t}^{a}-$ вероятность выявления и последующей изоляции, $B_{t}$ - количество новых зараженных, $\beta_{t}^{a}$ - заразность инфицированного, $I 0_{0}^{a}-$ распределение инфицированных по времени заражения в начальный момент времени. $n C_{t}$ - новые (выявленные) случаи заражения.

Непрерывная модель.

$$
\begin{aligned}
& \frac{\partial I}{\partial t}+\frac{\partial I}{\partial a}=-\lambda(a) I(t, a)-\mu(t, a) I(t, a) \\
& I(t, 0)=\int_{0}^{a_{\max }} \beta\left(t, a^{\prime}\right) I\left(t, a^{\prime}\right) d a^{\prime} ; \\
& I(0, a)=I_{0}(a)
\end{aligned}
$$

$\boldsymbol{S} \boldsymbol{I}_{15} \boldsymbol{R}-\boldsymbol{n C}$. Основная идея этой модификации модели $S I_{15} R$ состоит в мультипликативном представлении функций выявления и заразности:

$$
\begin{array}{ll}
\mu_{t}^{a}=I S_{t} \cdot m^{a} ; \quad & \sum_{\substack{a^{\prime}=0 \\
14}}^{14} m^{a^{\prime}}=1 \\
\beta_{t}^{a}=R 0_{t} \cdot b^{a} ; & \sum_{a^{\prime}=0}^{14} b^{a^{\prime}}=1
\end{array}
$$

где $I S_{t}$ - доля выявленных и изолированных от общего числа зараженных, $m^{a}-$ зависимость выявления от длительности заражения, $R 0_{t}$ - текущее репродуктивное контактное число (сколько в среднем заражает один инфицированный, при условии, что он не будет выявлен и изолирован), $b^{a}$ - заразность от длительности заражения.

Дополнительно, предполагаются достаточно очевидные ограничения на функции $\lambda^{a}, m^{a}$ и $b^{a}$ : 


$$
\begin{aligned}
& \lambda^{0}=0 ; \lambda^{1}=0 \\
& m^{0}=0 ; m^{1}=0 \\
& b^{0}=0 ; b^{1}=0 ; b^{14}=0
\end{aligned}
$$

и неубывание доли выявленных инфицированных

$$
I S_{t+1} \geq I S_{t} ; t \in[0, T] \text {, }
$$

отражающее увеличение усилий общества по выявлению и изоляции больных (в первые месяцы эпидемии).

\section{$S I_{15} R-n C-n T$}

Данная модификация модели связывает усилия общества по выявлению (и последующей изоляции) больных в зависимости от количества проведенных тестов.

$$
I S_{t}=I S_{n T}\left(n T_{t}\right)
$$

\section{$S I_{15} R-n C-n T-A n t i$}

Учет естественного иммунитета переболевших (естественного коллективного иммунитета)

$$
\begin{aligned}
& B_{t}=\left(1-A n t i_{t}\right) \cdot R 0_{t} \cdot \sum_{a^{\prime}=0}^{14} b^{a^{\prime}} I_{t}^{a^{\prime}} ; t \in[0, T] \\
& A n t i_{t}=1 / \mathrm{P}_{\text {mos }} \sum_{t^{\prime}=0}^{t} B\left(t^{\prime}\right)
\end{aligned}
$$

где $\mathrm{Anti}_{\mathrm{t}}$ - доля носителей антител в момент времени $t, P_{\text {mos }}-$ население Москвы.

\section{$S I_{15} R S-n C-n T-A n t i-I m$}

Учет уменьшения естественного коллективного иммунитета. 


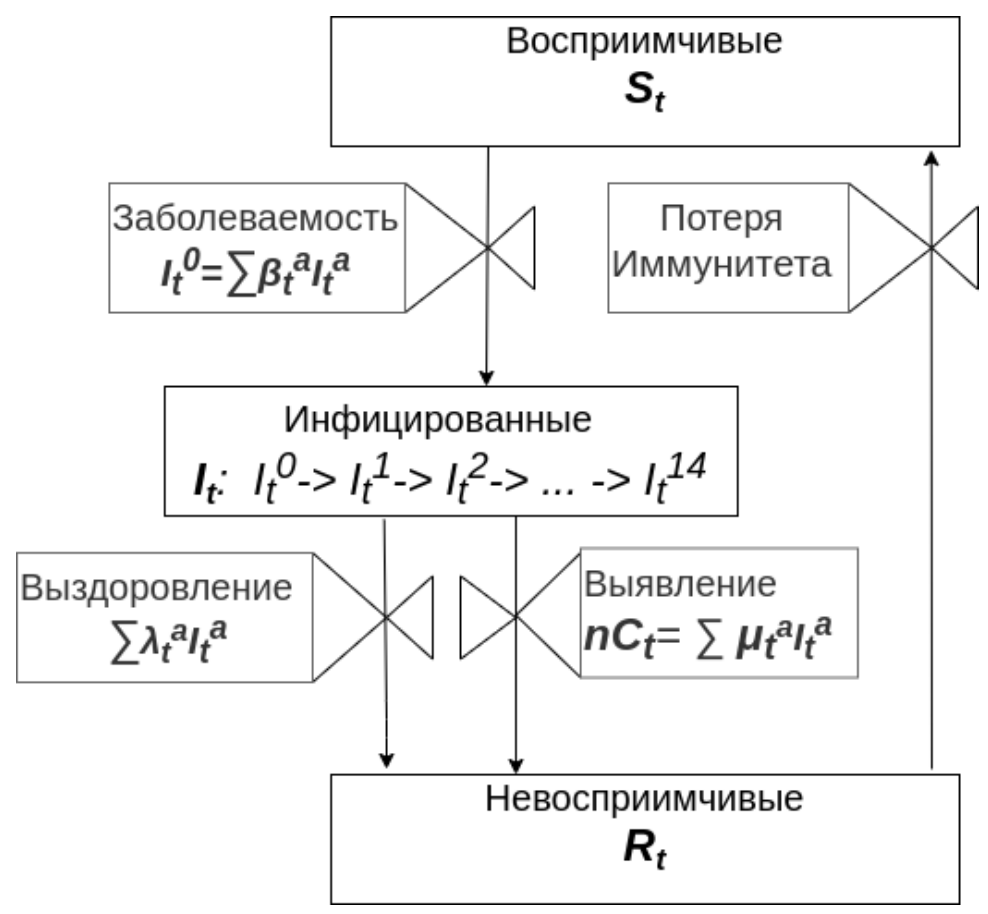

Рис. П2. Модель $\boldsymbol{S I}_{15} \boldsymbol{R S}$. Невосприимчивые (имеющие иммунитет) снова становятся восприимчивыми из-за потери иммунитета.

$A n t i_{t}=1 / P_{\text {mos }} \sum_{t^{\prime}=0}^{t}$ Imun $_{t-t^{\prime}} \cdot B_{t^{\prime}}$

где $\operatorname{Imun}_{\Delta t}$ - функция сохранения иммунитета как функция времени, прошедшего с момента заболевания $(\Delta t): 1$ - полный иммунитет (вероятность заболеть равна 0 ), 0 - полное отсутствие иммунитета.

\section{$S I_{15} R S-n C-n T-A n t i-I m-V u c$}

Учет вакцинации.

$$
B_{t}=\left(1-V u c c_{t-\delta}\right) \cdot\left(1-A n t i_{t}\right) \cdot R 0_{t} \cdot \sum_{a^{\prime}=0}^{14} b^{a^{\prime}} I_{t}^{a^{\prime}} ; t \in[0, T],
$$

где $\quad V_{u c c_{t}}-$ доля привитых к моменту $t$,

$\delta$ - запаздывание между временем прививки и началом ее действия (в расчетах используется величина 14 дней). 